\title{
IDENTIFICATION OF CHEMICAL REACTOR PLANT'S MATHEMATICAL MODEL
}

\author{
Boris Pyakillya ${ }^{1}$, Sergey Kladiev ${ }^{1 a}$ \\ ${ }^{1}$ National Research Tomsk Polytechnic University, 634050 Tomsk, Russia
}

\begin{abstract}
This work presents a solution of the identification problem of chemical reactor plant's mathematical model. The main goal is to obtain a mathematical description of a chemical reactor plant from experimental data, which based on plant's time response measurements. This data consists sequence of measurements for water jacket temperature and information about control input signal, which is used to govern plant's behavior.
\end{abstract}

\section{Introduction}

In current times in heating engineering there is an plants' optimal control problem which is connected with lack of well-tuning P, PI, PID controllers and others. One of the reasons is lack of insight into plants' behavior [1]. This can be solved only if engineers or scientists have accurate mathematical description of plants - transfer functions, differential of difference equations. Hence, system identification as a method to obtain mathematical models from experimental data has a great significance for optimal control problem and controller tuning problem. In a result researchers have to collect experimental data with ability to choose appropriate control input signals (active experiment) or use existing ones (passive experiment).

In most cases, researchers have an ability only to collect data from passive experiment because of impossibility to change the way of control processes which have high time delays [2].

Further, common identification problem description will be presented and also its mathematical description in terms of transfer function will be presented.

\section{Problem statement}

In this work chemical reactor's temperature control system is being investigated. The main control system's goal it to stabilize water temperature of cooling jacket using PID-controller. Schematic representation of chemical reactor is depicted below in Fig.1.

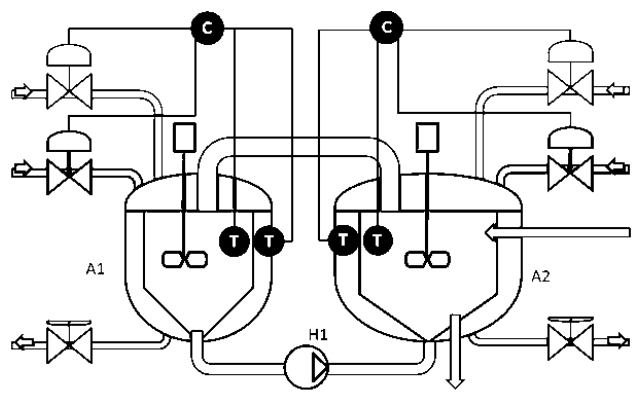

Figure 1. Scheme of chemical reactor

The problem is to get the mathematical description which is based on experimental data given from passive identification experiment. Experimental data is a sequence of cooling jacket temperature measurements $T_{p}$. Plot of temperature's transient process is depicted below in Fig.2. Units of y-axis are degrees Celsius, units of x-axis are seconds. Initial temperature is assumed as zero due to computational simplifications.

${ }^{\text {a }}$ Corresponding author : kladiev@tpu.ru 


\section{MATEC Web of Conferences}

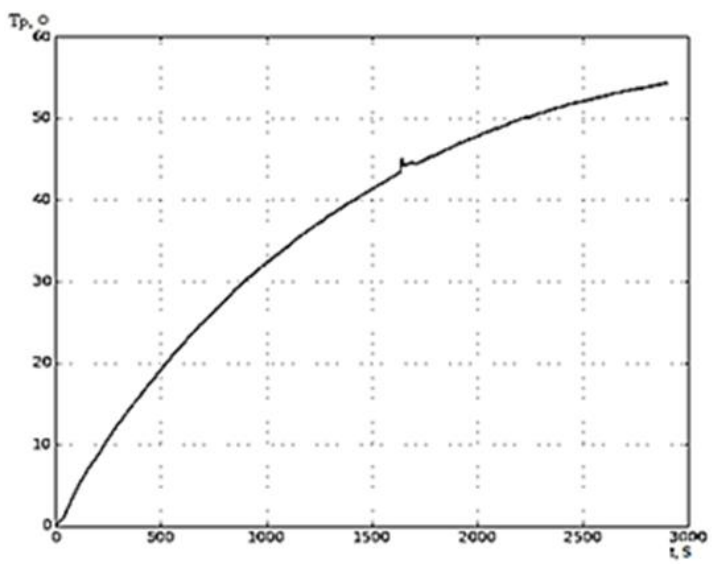

Figure 2. Temperature transient process of cooling jacket

Mathematical description based on this experimental data has to be obtained in terms of transfer functions due to engineering applications $[3,4]$. The structure of desired transfer function is

$$
W(s)=\frac{k}{a_{2} s^{2}+a_{1} s+1},
$$

where $s$-complex variable, $k$ - plant gain, $a_{2}, a_{1}$ - denominator coefficients of polynomial which describe physical properties of plant. Choice of particular structure (1) is based on transient process form, which has aperiodic behavior with small changes of second derivative value in initial time.

Obtained mathematical description should be verified through experimental data with help of some identification criterion. This criterion chosen as relative Chebyshev form expression:

$$
\Delta=\frac{\max _{1<i<N}\left|\mathrm{~T}_{\mathrm{p}}-\mathrm{T}_{\mathrm{M}}\right|}{\max _{1<i<N}\left(\mathrm{~T}_{\mathrm{p}}\right)} \cdot 100 \%,
$$

where $\mathrm{T}_{\mathrm{p}}, \mathrm{T}_{\mathrm{M}}$ - experimental measurements and model outputs, $N$-number of samples.

\section{Solution of the identification problem}

The identification problem consists estimation of transfer function (1) parameters and identification process should have low computational efforts. Chosen identification method is based on real interpolation method $[5,6]$ :

$$
F(\delta)=\int_{0}^{\infty} f(t) e^{-\delta t} d t, \delta \in[C, \infty), C \geq 0
$$

which used values of time function $f(t)$ for transfer to function of real variable $\delta$ as a real part of complex variable $s$. Expression (3) can be considered as a particular form of Laplace transform, where complex variable is replaced by its real part. Main property of these functions $\mathrm{F}(\delta)$ is that functions belong to real functions with real variable. It gives possibility to use well-developed methods of computational mathematics and optimization. It is important to notice that computational efforts get lower in comparison to frequency domain methods, which have to use steps of dividing a complex variable to real and imaginary parts.

Obtained mathematical model in terms of transfer functions presented below:

$$
W(s)=\frac{54,28}{15902,9 s^{2}+1081,8 s+1} .
$$

Identification criterion value (2) is $6,78 \%$, which tells about satisfactory fitting model output to experimental data. Plots of transient processes for plant and model correspondingly are depicted in Fig. 3. 


\section{Smart Grids 2015}

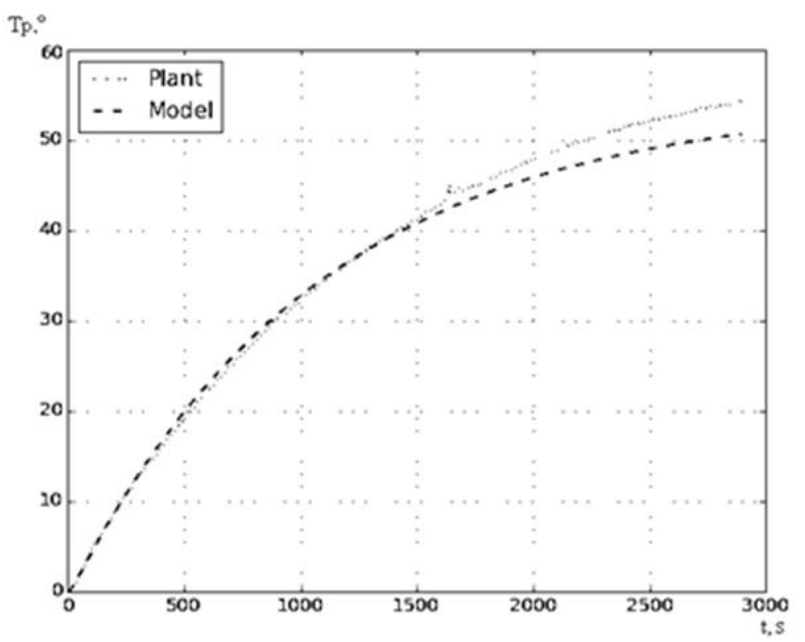

Figure 3. Plots of transient processes for plant and model

\section{Conclusion}

In result of this work, mathematical description of chemical reactor plant from experimental measurements was obtained. It gives the possibility to use it in optimal P, PI,PID-tuning for heat engineering control purposes.

The reported study was funded by RFBR according to the research project No. 15-38-50594 mol_nr.

\section{References}

1. Z. Labovská, J. Labovský, L. Jelemenský, J. Dudáš, J. Markoš, J. Loss Prev. Process Ind., 29, 155 (2014)

2. A.A. Shilin, V.G Bukreev, Automation and heat control in power engineering, 61, 741 (2014)

3. A.A. Shilin, V.G Bukreev, Thermal Engineering, 61 (9), 671 (2014).

4. K. A. Pupkov, N. D. Egupov, Mathematical Models, Dynamic Characteristics, and Analysis of Automatic Control Systems (Bauman MGTU, Moscow, 2004)

5. V. Goncharov, I. A. Aleksandrov, V. A. Rudnitsky, A.V. Liepinsh, Real Interpolation Method for Automatic Control Problem Solution (Saarbrucken : LAP LAMBERT Academic Publishing GmbH \& Co. KG, 2014)

6. V. Goncharov, A. Liepinsh, V. Rudnitskiy, Appl. Mech. Mater, 367, 312 (2013) 